\title{
Nanoscale
}

\section{Hierarchically Hydrogen-Bonded Graphene/Polymer Interfaces with Drastically Enhanced Interfacial Thermal Conductance}

Received 00th January 20xx, Accepted 00th January 20xx

DOI: $10.1039 / \times 0 \times x 00000 x$

www.rsc.org/

\author{
Lin Zhang, ${ }^{a}$ Ling Liu*a
}

Interfacial thermal transport is a critical physical process determining the performance of many material systems with smallscale features. Recently, self-assembled monolayers and polymer brushes have been widely used to engineer material interfaces presenting unprecedented properties. Here, we demonstrate that poly(vinyl alcohol) (PVA) monolayers with hierarchically arranged hydrogen bonds drastically enhance interfacial thermal conductance by a factor of 6.22 across the interface between graphene and poly(methyl methacrylate) (PMMA). The enhancement is tunable by varying the number of grafted chains and the density of hydrogen bonds in the unique hierarchical hydrogen bond network. The extraordinary enhancement results from a synergy of hydrogen bonds and other structural and thermal factors including molecular morphology, chain orientation, interfacial vibrational coupling and heat exchange. Two types of hydrogen bonds, i.e. PVAPMMA hydrogen bonds and PVA-PVA hydrogen bonds, are analyzed and their effects on various structural and thermal properties are systematically investigated. These results are expected to provide new physical insights for interface engineering to achieve tunable thermal management and energy efficiency in a wide variety of systems involving polymers and biomaterials.

\section{INTRODUCTION}

Interfacial thermal transport plays a critical role in determining the thermal performance of many innovative material systems with small-scale features, such as nanocomposites, nanoelectronics and some biomedical devices ${ }^{1-8}$. Typically, the interface between dissimilar materials inhibits transmission of heat energy across materials boundaries, making it a bottleneck for thermal transport especially when the characteristic length of the structure is comparable to the mean free path of energy carriers. ${ }^{9}$ For instance, the interface between graphene and polymers has low thermal conductance of $60 \sim 80 \mathrm{MW} \mathrm{m}^{-2} \mathrm{~K}^{-1}, 10-$ 12 making it far less conductive than graphene itself. This is believed to be the primary reason why graphene/polymer composites have thermal conductivities lower than predictions. Hence, improving interfacial thermal transport between materials of drastically different properties has been an important area of research seeking enhanced thermal management and energy efficiency.

Theoretically, phonon transmission across an interface strongly depends on the interfacial bonding strength as well as the coupling in vibrational modes between two materials

\footnotetext{
a. Department of Mechanical and Aerospace Engineering, Utah State University Logan, UT 84322, USA. *E-mail: ling.liu@usu.edu

†Electronic Supplementary Information (ESI) available: System setup for nonequilibrium molecular dynamics simulation system, heat energy added to the heat source in NEMD, temperature contour and atomic number density of G-32PVA temperature profiles from NEMD, axial atomic number density distribution, local Herman's orientation factors, criterion of hydrogen bonds, distribution and number of hydrogen bonds, system setup for NEMD pull-out simulation. See DOI: $10.1039 / x 0 x \times 00000 x$
}

forming the interface. ${ }^{9,13}$ On one hand, parametric studies by molecular dynamics simulations with Lennard-Jones potentials have shown that increasing the interfacial bonding strength significantly enhances interfacial thermal conductance between graphene and paraffin ${ }^{11}$ and between silicon and amorphous polyethylene. ${ }^{14}$ According to phonon wave packet simulation, these improvements are mainly caused by the higher transmission coefficients of both longitudinal and transverse acoustic waves and the broadened frequency spectrum of efficiently transmitted phonons. ${ }^{14}$ On the other hand, diffuse mismatch theory states that larger overlap of phonon states between two materials may cause higher probability of phonon transmission across their interface leading to higher interfacial thermal conductance. ${ }^{7,15}$ Recent studies have further concluded that interfacial thermal transport is dictated by the overlap of on-site vibrational modes between materials right at the interface, ${ }^{15,16}$ whereas high overlap between materials in the bulk form does not necessarily lead to high interfacial thermal transport.

One solution to improve interfacial thermal transport is to add an intermediate layer of a third material considering both of the interfacial bonding strength and vibrational mode coupling. However, achieving both objectives in one design is not straightforward and could be very challenging. For example, using polymer molecules as the vibrational linker was found to improve interfacial thermal transport between graphene and polymers by about $30 \%$ only. ${ }^{17,18}$ The improvement was limited mainly due to the weak van der Waals interaction on both sides of the added vibrational linker. Incorporating the much stronger covalent bond may solve this problem. Several studies have been reported recently to enhance thermal transport across metal/dielectric, ${ }^{19,20}$ carbon nanotube 
arrays/metal, ${ }^{21}$ graphene/metal ${ }^{22}$ and metal/metal ${ }^{23}$ interfaces by engineering covalent bonds on both sides of the added intermediate layer. However, application of this treatment is severely restricted by the few choices of materials that have the required bonding capability. Many efforts have then been made to search for better intermediate layers ${ }^{24,25}$ with balanced effectiveness and design flexibility. ${ }^{26}$ Recently, the hydrogen bond, a strong and important secondary bond that ubiquitously exists in many natural and synthetic materials, has been used to improve thermal transport across graphene/polymer ${ }^{27}$ and self-assembled monolayer/organic liquid 28 interfaces. Hydrogen bonds have been experimentally demonstrated to improve the thermal conductivity of polymers by blending polymer molecules with hydrogen bond moieties. ${ }^{29-31}$ For instance, a network of strong hydrogen bonds improves the thermal conductivity by an order of magnitude for a polymer blend compared to its constituents, poly( $\mathrm{N}$-acryloyl piperidine) (PAP) and poly(acrylic acid) (PAA). ${ }^{31}$ Besides, hydrogen bonding between poly(vinyl alcohol) (PVA) and short chains of diethylene glycol (DEG) can form a polymer blend of thermal conductivity $175 \%$ and $260 \%$ higher than that of PVA and DEG, respectively. ${ }^{29}$ Despite the progress, little attention has been paid to the structure of hydrogen bonds especially on interfacial thermal transport. With hydrogen bonds structurally arranged between dissimilar materials, a hydrogen bond network has the potential to drastically improve interfacial thermal conduction through novel energy transport mechanisms.

This study probes new energy transport mechanisms when hierarchically arranged hydrogen bonds are designed to decorate graphene/polymer interfaces for highly efficient thermal bridging. The hierarchical hydrogen bond network is enabled by covalently attaching polymer chains onto graphene with controllable density and orientation leading to the socalled polymer brush or the self-assembled monolayer. This is experimentally achievable by various methods including atomic transfer radical polymerization, ${ }^{32,33}$ irradiation-induced polymerization, ${ }^{34}$ ring-open metathesis polymerization, ${ }^{35}$ thiol- ene click reactions ${ }^{36}$ and esterification/amidation reactions. ${ }^{37,38}$ Hydrogen bonds form not only between the polymer monolayer and the polymer matrix, but also exist between neighboring polymer chains within the monolayer. We demonstrate that this design significantly enhances interfacial thermal conductance by $622 \%$, mainly due to hydrogen-bond-enabled thermal transport pathways. Effects of grafting density, chain conformation, and hydrogen bond density are systematically investigated. The results will guide materials design in nanocomposites as well as hybrid biomaterials and energy materials for tunable thermal management and energy efficiency.

\section{Models and Methods}

\subsection{Models}

Full atomic models of nanocomposite interfaces were built by combining poly(vinyl alcohol) (PVA)-functionalized graphene and a poly (methyl methacrylate) (PMMA) matrix, as shown in Fig. 1(a). PVA molecules are covalently grafted onto graphene. Each PVA chain has eight repeat units of $-\mathrm{CH}_{2}-\mathrm{CH}(\mathrm{OH})$. Note that length of PVA chains is expected to influence interfacial thermal conductance as it influences the number of hydrogen bonds and PVA configurations. However, to keep the research more focused, length effect was neglected and eight repeat units were used in all models for the parametric study and mechanism investigations. All PVA chains are uniformly distributed on graphene. The relaxed PVA-grafted graphene has a cross-sectional area of $34.45 \AA \times 34.10 \AA$, large enough to capture dominant vibrational modes of interfacial thermal conduction. ${ }^{11}$ The PMMA matrix was built by the self-avoiding random walk method, ${ }^{39}$ with 40 repeat units per chain. Both material models were generated by our in-house codes, well equilibrated separately, and then merged in $\mathrm{VMD}^{40}$ to form the

(a)

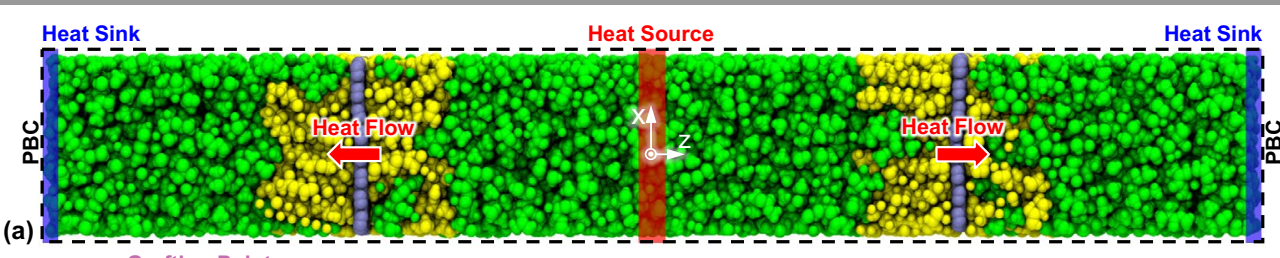

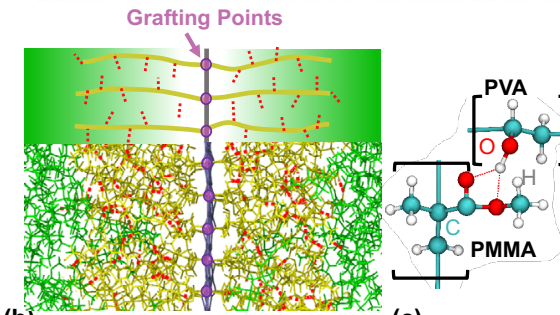

(b)

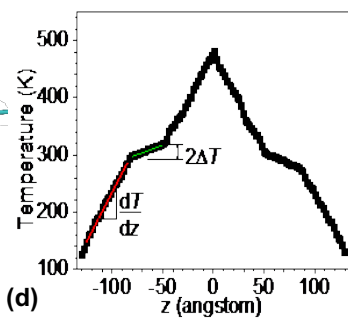

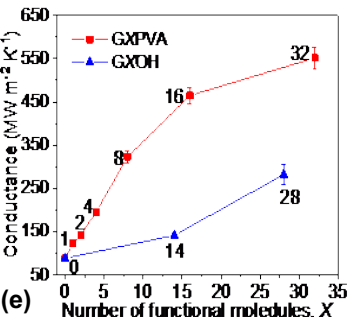

Fig. 1. (a) A full-atom model of the interface between PMMA (green spheres) and a graphene monolayer (ice blue spheres) functionalized by PVA chains (yellow spheres). (b) 3D hydrogen bond network at the interface of G-32PVA (32 PVA chains are grafted onto each side of the graphene monolayer). A schematic (top right 3/8) and an MD snapshot (bottom right $5 / 8$ and left part) show how hydrogen bonds (red dashed lines) are hierarchically arranged. A repeat unit of PVA and PMMA depicts the hydrogen bond formation. Both oxygen atoms of ester group in PMMA can be hydrogen bond acceptors. (c) An illustration of hydrogen bonds (red dash lines) between the repeat units of PVA and PMMA. Spheres in red, green and white represent oxygen, carbon, and hydrogen atoms, respectively. (d) Temperature profile generated by reverse nonequilibrium molecular dynamics (RNEMD) simulation for G-32PVA. Total temperature drop across the interface is denoted by $2 \Delta T$. Temperature gradient of PMMA (denoted by $\mathrm{d} T / \mathrm{d} z$ ) is found by curve fitting. (e) Interfacial thermal conductance versus the number of functional molecules grafted on graphene for G-XPVA, G-XPE and G-XOH, ${ }^{26}$ respectively. 
nanocomposite interface. Periodic boundary conditions were applied in all three directions. The formed nanocomposite interface is denoted as "G-XPVA" for brevity, where " $G$ " denotes graphene and $X$ is the number of PVA chains on each side of graphene in the present unit cell model. The effect of chain density is studied by varying the value of $X$. Hydrogen bonds form between PVA and PMMA (see Fig. 1(c)), and between densely packed PVA chains as well (see Fig. 1(b) for an example of G-32PVA).

\subsection{Methods}

Molecular dynamics (MD) simulations were carried out using LAMMPS. ${ }^{41}$ Interatomic interactions were described by the OPLSAA force field, ${ }^{42,43}$ which has been widely used to simulate polymers, graphene, and their interfaces. ${ }^{17,25,44,45}$ Four steps were taken to prepare nanocomposite interfaces for production runs. First, the initial atomic model of PMMA was optimized by the conjugate gradient algorithm, and then equilibrated in the NPT ensemble at $300 \mathrm{~K}$ and $1.0 \mathrm{~atm}$ for $1.0 \mathrm{~ns}$, and finally equilibrated in the NVT ensemble at $300 \mathrm{~K}$ for $1.0 \mathrm{~ns}$. Second, atomic models of nanocomposite interfaces were generated by sandwiching PVA functionalized graphene in between the PMMA matrix generated from the first step. Third, nanocomposite interfaces were annealed in the NPT ensemble at $1.0 \mathrm{~atm}$ to remove residual stresses. During annealing, the system was heated up from $300 \mathrm{~K}$ to $800 \mathrm{~K}$, maintained at 800 $\mathrm{K}$ for $500 \mathrm{ps}$, and then cooled down to $300 \mathrm{~K}$ at a rate of 1.667 $\mathrm{K} / \mathrm{ps}$. After that, the system was relaxed in NPT and NVT for 500 ps, respectively. In all of these steps, time step was set to be 1.0 fs. Lastly, additional relaxation was performed in NVT at $300 \mathrm{~K}$ for $1.0 \mathrm{~ns}$ with the time step of $0.5 \mathrm{fs}$. In all production runs, time step was fixed at $0.5 \mathrm{fs}$ which is short enough to capture vibrations of hydrogen atoms.

Reverse non-equilibrium molecular dynamics (RNEMD) proposed by Muller-Plathe ${ }^{46}$ was used to calculate interfacial thermal conductance, as illustrated in Fig. 1(a). Interfacial thermal conductance $(G)$ was calculated by $G=J / \Delta T$, where $J$ is the heat flux imposed by numerical manipulation and $\Delta T$ is the temperature drop evaluated across the interface. As shown in Fig. 1(a), the entire system was evenly divided into 100 slabs in the $z$-direction. A certain amount of heat energy was added to the heat source (the red slab), while the same amount of energy was subtracted from the heat sink (the blue slab). This manipulation was achieved by velocity swapping with a virtual elastic collision model ${ }^{47}$ to maintain momentum conservation. As a result, a heat flow was generated from the heat source to the heat sink along both $+z$ and $-z$ directions. Numerically, heat flux was calculated by $J=\Delta E /(2 t A)$, where $\Delta E$ is the energy added to the heat source during the time of $t, A$ is the crosssectional area of the system, and " 2 " accounts for the two symmetric heat transport paths from heat source to heat sink. As illustrated in Fig. 1(b), temperature drop across the interface was denoted by $2 \Delta T$, which was controlled to be $45 \pm 5 \mathrm{~K}$ by tuning the frequency of velocity swapping. In addition, thermal conductance of PMMA was calculated by $k=J /(d T / d z)$, where

This journal is (C) The Royal Society of Chemistry 20xx $d T / d z$ is the temperature gradient of PMMA, as shown in Fig. 1 (d).

To visualize temperature contours at the interface, we performed nonequilibrium molecular dynamics simulation (NEMD) by fixing temperatures of the heat source and the heat sink at $400 \mathrm{~K}$ and $250 \mathrm{~K}$, respectively. Temperature was controlled by the Langevin thermostat ${ }^{48}$ in NVE. As shown in Fig. S1, atoms of the right part of the system were fixed during the simulation. After a steady state was reached, atomic temperatures and positions were collected during a run of 5.0 ns. Atoms were binned into $80(y) \times 560(z)$ cells and pointwise temperature was obtained by averaging atomic temperatures in each cell.

\section{RESULTS AND DISCUSSION}

\subsection{Validation}

To validate computational models and methods, we compared our MD results with experimental and computational data from the literature focusing on four properties. First, mass density of the PMMA matrix far away from the interface in well relaxed simulation systems was calculated to be $1.14 \pm 0.03 \mathrm{~g} \mathrm{~cm}^{-3}$, well within the range of experimental data, $1.1 \sim 1.19 \mathrm{~g} \mathrm{~cm}^{-3} .45$ Second, interfacial thermal conductance of G-32PVA was found to be $551.53 \pm 24.81 \mathrm{MW} \mathrm{m}^{-2} \mathrm{~K}^{-1}$ and $570.83 \mathrm{MW} \mathrm{m}^{-2} \mathrm{~K}^{-1}$ as calculated by RNEMD and NEMD, respectively, which agree well with each other. Third, interfacial thermal conductance of the pristine graphene/PMMA interface was evaluated as $88.66 \mathrm{MW}$ $\mathrm{m}^{-2} \mathrm{~K}^{-1}$, within the range of $60 \sim 150 \mathrm{MW} \mathrm{m}^{-2} \mathrm{~K}^{-1}$ for similar pristine graphene/polymer interfaces. ${ }^{10-12}$ Lastly, thermal conductivity of the PMMA matrix was calculated to be 0.20 $\pm 0.01 \mathrm{~W} \mathrm{~m}^{-1} \mathrm{~K}^{-1}$, which is also in good agreement with experimental values from 0.19 to $0.25 \mathrm{~W} \mathrm{~m}^{-1} \mathrm{~K}^{-1} .49,50$

\subsection{Effect of grafting density on interfacial thermal conductance}

Fig. 1(e) shows that interfacial thermal conductance of G-XPVA increases significantly when more PVA chains are grafted on graphene. Here, the number of PVA chains on each side of graphene, $X$, ranges from 0 to 1, 2, 4, 8, 16 and 32. Notably, as the PVA coverage on graphene increases to $7.14 \%$ (i.e. G32PVA), interfacial thermal conductance is improved to be 6.22 times that of the pristine graphene/PMMA interface (551.53

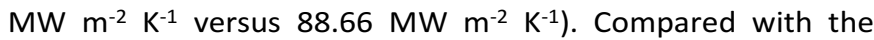
previously studied hydroxyl-functionalized graphene/PMMA interface which features a $2 \mathrm{D}$ hydrogen bond layer, ${ }^{27}$ the present system with a 3D hierarchical hydrogen bond network improves the interfacial thermal conductance further by 1-2 times, especially when $X<16$. As demonstrated in Fig. 1(e), even two PVA chains outperform fourteen hydroxyl groups in thermal conduction enhancement. Note that, structurally, there is a key difference between the two systems: heat flow is parallel to hydrogen bonds in the $\mathrm{OH}$-grafted system, but mostly perpendicular to hydrogen bonds in the PVA-grafted system. Moreover, this comparison is based on the number of functionalization sites on graphene. There is also another measure that can be used for comparing thermal enhancement 


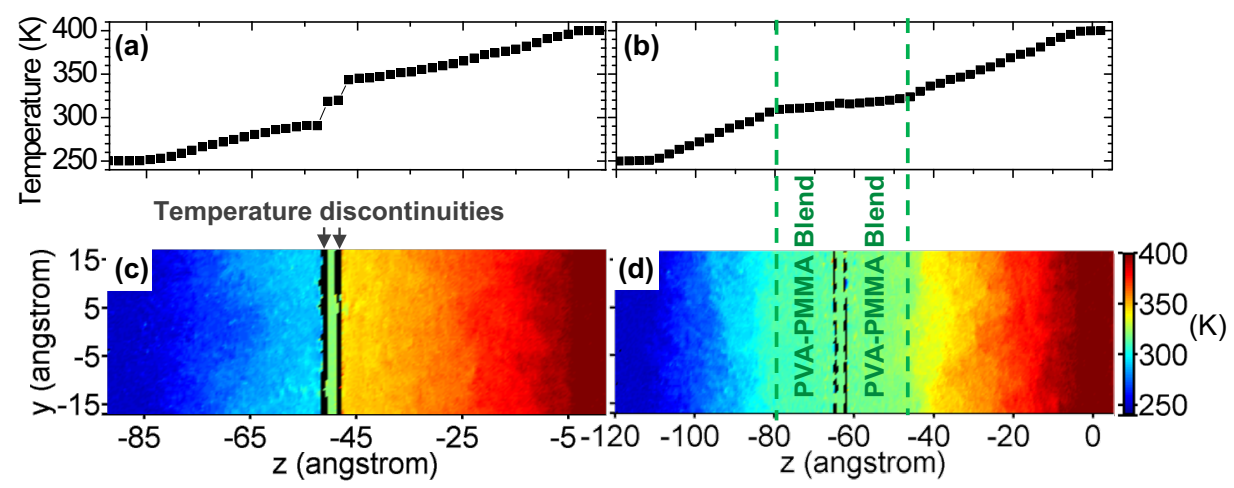

Fig. 2. Temperature profiles of (a) G-1PVA and (b) G-32PVA. Temperature contours of (c) G-1PVA and (d) G-32PVA. Two vertical green lines are added in (b) and (d) to show the PVA-PMMA blending region. Black stripes in (c) and (d) indicate regions that are unoccupied by any atoms due to steric repulsion.

between different systems, i.e. the number of hydrogen bonds. Its effect is discussed in Section 3.5, where the contributions of both the backbone and the hydrogen bonds formed at side chains are quantified for the present hierarchical design.

The improved interfacial thermal transport performance is also evidenced by Fig. 2, which depicts the temperature profiles and contours of both G-1PVA and G-32PVA. With more PVA chains present at the interface, G-32PVA demonstrates much smoother temperature variations with alleviated discontinuities in the vicinity of the interface. Here, temperatures at the two ends of both systems were fixed at $400 \mathrm{~K}$ and $250 \mathrm{~K}$, respectively. The required heat flux in G-32PVA is $20 \%$ higher than that of G-1PVA (Fig. S2) due to the thermally more efficient interface. For G-1PVA which has sparse PVA chains, an abrupt temperature drop across the interface is identified in Fig. 2(a), indicating a large thermal resistance and poor interfacial thermal transport. Accordingly, Fig. 2(c) shows a sharp change in color from dark red to deep blue across the interface. The two black stripes in Fig. 2(c) between graphene and PMMA are zones unoccupied by any atoms due to steric repulsion. The discontinuities adversely influence thermal transport across the interface. By contrast, G-32PVA does not show any abrupt temperature drop (Fig. 2(b)). The region by two green lines in Fig. 2(b) features a blend of PVA and PMMA with densely packed PVA chains, where temperature drop is small (Fig. 2(d)). Moreover, due to more covalently bonded PVA chains in G32PVA, some of the discontinuities previously seen in G-1PVA are alleviated or removed. As shown in Fig. 2(d), the discontinuity strips become thinner and they are broken into pieces at the PVA-grafting points where PVA chains are anchored to graphene (Fig. 1(b)). These newly established thermal transport pathways make interfacial heat transfer more efficient in G-32PVA. The improved interfacial thermal transport can also be seen in the temperature contours plotted in Fig. 3(a)-(b) for individual PVA chains (see Fig. S3 for more plots of G-32PVA). Under the same $\triangle T$ across the systems, PVA chains in G-32PVA show much smaller temperature drop than those in G-1PVA, indicating fast thermal transport.

\subsection{Effect of grafting density on interfacial molecular morphology and hydrogen bonds}

Molecular morphology significantly affects thermal transport in polymers at the nanoscale. Extended polymer chains and nanofibers exhibit thermal conductivities several orders of magnitude higher than their amorphous counterparts. ${ }^{51-53}$ It is well known that self-assembled monolayers usually lie flat on the substrate at low coverage densities; and they "stand up" to form a "quasi-crystalline" structure when surface saturation is reached. 54,55

However, contrary to the conventional wisdom, PVA chains in this study are found to have the "stand up" configuration even at very low grafting densities. Fig. 3(c)-(d) plot the atomic number density of PVA and graphene in G-1PVA and G-32PVA,

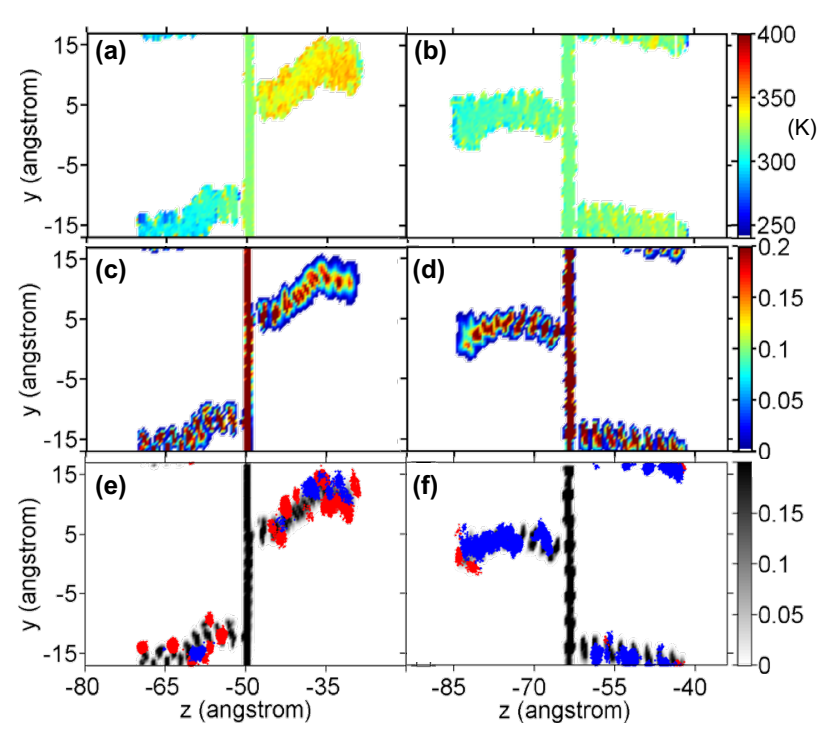

Fig. 3. Temperature contours $(a, b)$, atomic number density distributions (c, d) and hydrogen bond distributions (e, f) in G-1PVA and G-32PVA, respectively. Only graphene and a pair of PVA chains are shown for clarity. The pair of PVA chains shown for G-32PVA were selected from a total of 32 pairs. Each dot in (e) and (f) represents a hydrogen bond using the position of its hydrogen atom. Red dots are hydrogen bonds formed between PVA and PMMA, while blue dots represent those formed within or between PVA chains. Gray scale bar in $(e, f)$ shows the atomic number density. 


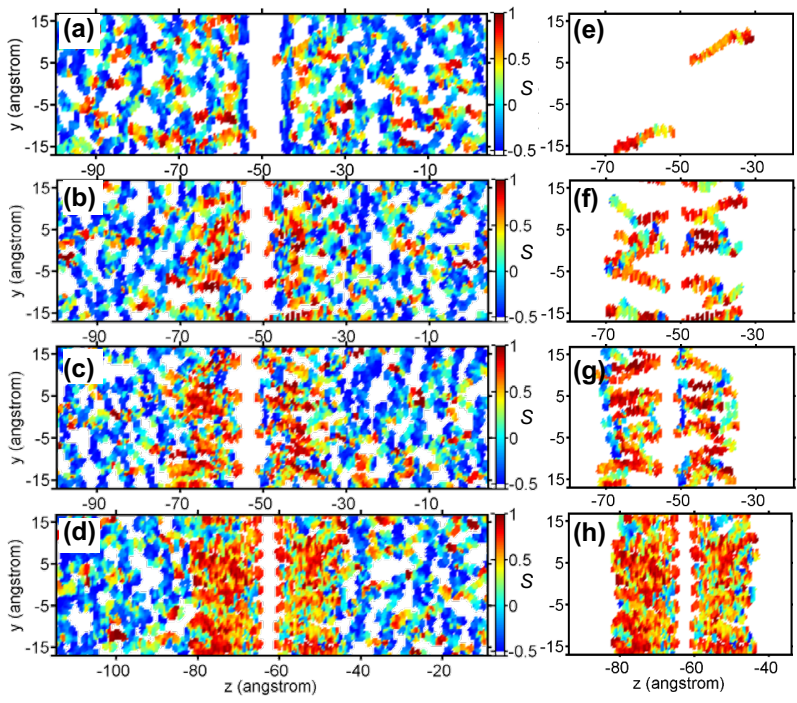

Fig. 5. Local Herman's orientation factor for the PMMA matrix with the PVA laye (left) and PVA only (right) in G-XPVA where $X=1$ (a, e), 8 (b, f), 16 (c, g), and 32 (d, h). Atomic coordinates were collected by equilibrium MD simulations over $10.0 \mathrm{~ns}$ in NVE.

respectively, where only graphene and a pair of PVA chains are shown for clarity. The two PVA chains shown for G-32PVA are selected from the 64 chains grafted on both sides of graphene. Plots for another four pairs can be found in Fig. S4. All results suggest extended configurations even at very low grafting densities (e.g. G-1PVA), although they are not as extended as those in the densely packed G-32PVA. Similar conclusions can also be drawn from axial density distribution results of PVA (Fig. S5).

To further verify the extended configurations of PVA chains, Herman's orientation factor was evaluated for PVA and PMMA in the vicinity of graphene. Widely used to quantify polymer chain orientations, Herman's factor ${ }^{56}$ is defined by $S=1.5$ $<\cos 2 \theta>-0.5$, where $\theta$ is the angle of a polymer chain with respect to a predefined reference orientation, and <-> represents a spatial average over the entire or a part of the system. Particularly, $\mathrm{S}=1$ means that all polymer chains are perfectly aligned with the reference orientation, while $S=0$ suggests an amorphous polymer. In this study, polymer atoms were binned into $80 \times 560$ cells in the $y-z$ plane; and $S$ was evaluated in each cell using $\mathrm{z}$-axis as the reference orientation. The $S$ values evaluated for PVA and PMMA are plotted in Fig. 4 for systems with different numbers of PVA chains. The blank region in the middle of all subfigures is where graphene resides. Red blobs near graphene correspond to the ends of PVA chains where they are grafted onto graphene (i.e. grafting points). Blue blobs mostly correspond to PMMA as its chains are randomly orientated. Red blobs are closer to graphene than blue blobs due to the covalent bonding between PVA and graphene. Therefore, as more PVA chains are grafted on graphene, more red blobs are present making the blank region appear to decrease in width. Additionally, Fig. S6 shows that the gap between PMMA on the two sides of graphene increases with the PVA grafting density. Tracing from the grafting points, PVA chains in all cases are found mostly in red or orange, indicating

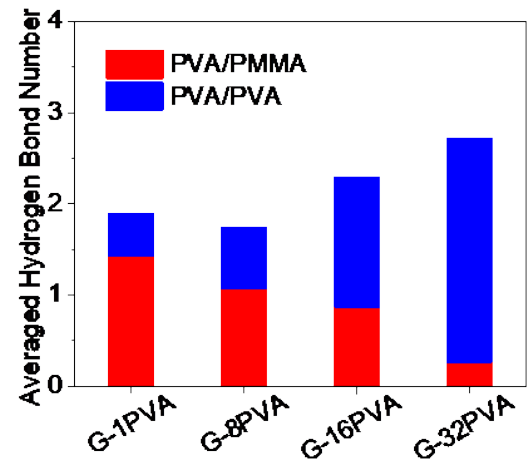

Fig. 4. Averaged number of hydrogen bonds per PVA chain for systems of different grafting densities. Red shows hydrogen bonds formed between PVA and PMMA, while blue shows those formed within or between PVA chains. Results are obtained from equilibrium MD simulations of over 60 ps.

$\theta$ close to $0^{\circ}$ (almost perpendicular to graphene suggesting a "stand-up" configuration). As more PVA chains are grafted at the interface, less PMMA chains enter the blend region and those in the region show extended configurations as well (see Fig. S6). The results suggest a blend region full of extended chains (mostly PVA and some PMMA) for G-32PVA.

The PVA chains are highly extended mainly due to their strong affinities with the surrounding PMMA matrix as a result of the hierarchical hydrogen bond network. Fig. 3(e)-(f) depict the distribution of hydrogen bonds over 10000 output frames by averaging the results of different runs in NVE ensemble for each system. Here the time step is $0.2 \mathrm{fs}$ and the output frequency is $2 \mathrm{fs}$. A red dot represents a hydrogen bond between PVA and PMMA, while a blue dot represents a hydrogen bond between hydroxyl groups within a PVA chain or between neighboring PVA chains. The criteria used to identify a hydrogen bond are described in Fig. S7.

As shown in Fig. 3(e) for G-1PVA, at the low PVA grafting density, more hydrogen bonds form between PVA and PMMA than within PVA; and hydrogen bonds between PVA and PMMA are well distributed along the PVA chains. The extended configuration allows larger exposure of PVA chains to PMMA, leading to more hydrogen bonds and making the structure energetically more favorable than other configurations.

As shown in Fig. 3(f) for G-32PVA, with its high PVA grafting density, intra-PVA hydrogen bonds dominate, while hydrogen bonds with PMMA mainly form at the free ends of PVA chains (see Fig. $\mathbf{5 8}$ for more examples). The unique hydrogen bond structure along with steric repulsion makes PVA chains highly extended at high grafting densities.

Fig. 5 shows the number of hydrogen bonds per PVA chain at different PVA grafting densities. The number of hydrogen bonds between PVA and PMMA (red) decreases with the number of PVA chains, while the number of intra-PVA hydrogen bonds (blue) increases. G-32PVA is found to possess the most hydrogen bonds per PVA chain with both types combined. Given that it also has the most PVA chains, the total number of hydrogen bonds in G-32PVA is far beyond those in other systems under investigation (Fig. S9). Due to the importance of hydrogen bonds in nanoscale thermal transport, ${ }^{57-61}$ the vast 


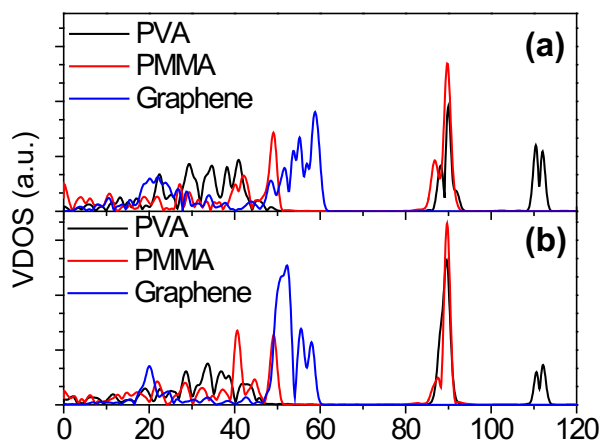

Fig. 7 Partial vibrational density of states (VDOS) of (a) G-1PVA and (b) G-32PVA. VDOS is displayed for three groups, namely, PVA, PMMA, and the sp2 carbon atoms of graphene.

number of hydrogen bonds available in the present nanocomposite system should enhance thermal transport performance.

\subsection{Mechanisms of thermal enhancement}

Mechanisms of thermal enhancement enabled by PVA decoration (Fig. 1(e)) are examined from two perspectives, i.e. interfacial vibrational coupling and interfacial adhesion. Material interfaces are in general less thermally conductive when the two materials have low similarity in vibrational density of states (VDOS). VDOS is usually computed by Fourier transformation of the velocity autocorrelation function. To focus on the coupling of on-site vibrational modes, VDOS was generated for materials right at the interface, not in their bulk forms. Atomic velocities were sampled every $2.0 \mathrm{fs}$ based on equilibrium MD simulations. A correlation factor $(M)^{25,62}$ is then used to quantify the similarity in on-site VDOS between the two materials.

$$
M=\frac{\int_{0}^{\infty} \operatorname{VDOS}_{A}(f) \cdot \operatorname{VDOS}_{B}(f) \mathrm{d} f}{\int_{0}^{\infty} \operatorname{VDOS}_{A}(f) \mathrm{d} f \cdot \int_{0}^{\infty} \operatorname{VDOS}_{B}(f) \mathrm{d} f}
$$

where subscripts $A$ and $B$ denote the two materials comprising the interface. Large $M$ indicates better overlap in vibrational modes between materials.

Adding PVA to graphene is found to enhance vibrational coupling at graphene/PMMA interfaces as evidenced by Fig. 6, which plots vibrational modes in three groups (namely, PVA, PMMA and $s p^{2}$ carbon atoms of graphene) for G-1PVA and G32PVA, respectively. Firstly, as more PVA chains are grafted on graphene, more $s p^{2}$ carbon atoms become $s p^{3}$ carbon at the grafting points, broadening the peak at $51.5 \mathrm{THz}$ for G-32PVA. This vibrational mode has a good overlap with PMMA as shown in Fig. 6(b). Secondly, PMMA has vibrational modes around 89.9 $\mathrm{THz}$, which have no overlap with graphene but is coincident with that of PVA. For these reasons, G-32PVA shows improved vibrational coupling at the interface. The $M$ factor between PVA and PMMA is calculated to be 0.0376 in G-32PVA, higher than 0.0328 in G-1PVA; and the $M$ factor between graphene and PMMA is calculated to be 0.0099 in G-32PVA, also higher than 0.0084 in G-1PVA.

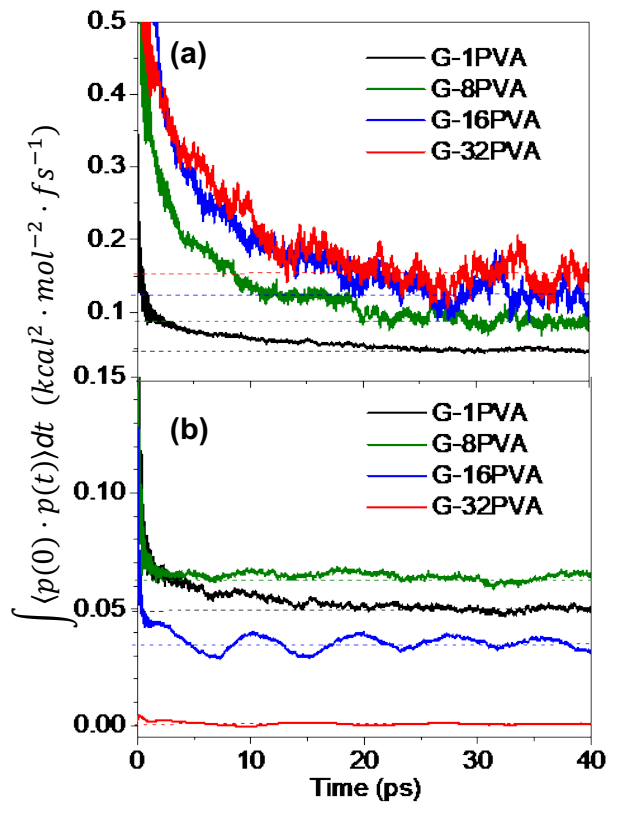

Fig. 6 Integrated autocorrelation of interfacial heat power (a) between PVA and PMMA and (b) between graphene ( $s p^{2}$ carbon) and PMMA. Dashed lines show converged values of the integral.

Furthermore, the PVA/PMMA interface which is more present in G-32PVA shows much higher coupling than graphene/PMMA which is more present in G-1PVA ( $M=0.0376$ versus 0.0084). As a result, grafting PVA chains improves interfacial thermal conductance, with more heat conducted through the highly efficient PVA-PMMA channel; while at low grafting densities, most heat is conducted through the less efficient graphene-PMMA channel. A direct proof of this conclusion can be obtained by computing integrated autocorrelation of interfacial heat power ${ }^{27}$ which is proportional to interfacial thermal conductance according to Green-Kubo fluctuation theorem. Fig. 7 plots integrated autocorrelation of interfacial heat power between PVA and PMMA and between graphene and PMMA, respectively, at four PVA grafting densities. It is apparent that, as more PVA chains are grafted, more heat is conducted through the highly efficient PVA-PMMA channel, enhancing the overall interfacial thermal transport performance.

It is worth noting that the VDOS study discussed above focuses on non-bonded molecular pairs at the interface only. VDOS coupling between the bonded pair, i.e. graphene and PVA, is not investigated because bonded structures are usually better conductors than nonbonded molecular pairs. The present work aims to improve interfacial thermal transport by enhancing the nonbonded pairs which are the "bottleneck".

Another way to understand interfacial thermal enhancement is to correlate interfacial conductance with interfacial adhesion. In general, higher adhesion presents better thermal conduction. ${ }^{20,63-65}$ Different from vibrational modes which represent bonding stiffness at equilibrium, interfacial adhesion describes the interfacial bonding strength and the affinity between materials. 66 


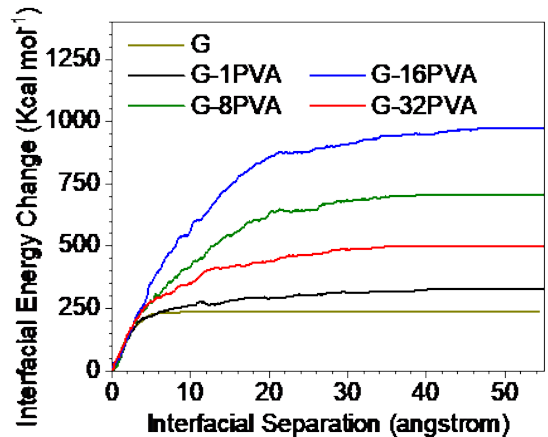

Fig. 8. Interfacial energy change versus interfacial separation for G-XPVA with different PVA grafting densities. "G" in the plot represents the case with the pristine graphene/PMMA interface.

Fig. 8 plots variations of interfacial energy as pristine and PVA-functionalized graphene are pulled apart from the PMMA matrix by MD simulations as described in Fig. S10. In all cases, interfacial energy between the PMMA and graphene increases as these two phases are separated. The converged values show the affinity energy or work of adhesion. Due to the hierarchical hydrogen bond network, work of adhesion increases with the number of PVA chains when $X \leq 16$, echoing the enhancement of interfacial thermal conductance with more PVA chains. However, G-32PVA is an exception. It has the highest interfacial conductance but relatively low affinity energy.

In G-32PVA, PVA chains are so dense that they form a PVA layer with few PMMA chains in it. PMMA chains are expelled from the blending region, and they form a relatively planar interface with the PVA layer. The structural change affects hydrogen bonds formation (Fig. 5), reducing the number of hydrogen bonds between PVA and PMMA and increasing that between PVA chains. Most of the PMMA-PVA hydrogen bonds now form at a relatively planar interface between the densely packed PVA layer and the PMMA (Fig. 3(f)).

Thermal transport becomes more efficient in G-32PVA for three reasons. First, PVA chains are more extended due to intraPVA hydrogen bonds and steric repulsion between neighboring PVA chains, which is a positive factor for along-chain thermal transport. Second, with a mechanism similar to that previously shown by us for protein $\beta$-sheets and polymer nanofibers, ${ }^{59-61}$ intra-PVA hydrogen bonds can enhance thermal transport across the densely packed PVA layer. Third, the hydrogen bonds formed between PVA and PMMA facilitate thermal transport across the relatively planar interface.

Despite the higher interfacial thermal conductance in G32PVA, interfacial bonding force (see Fig. S11) and adhesion of work both drop compared with G-16PVA. The primary reason is the structural change from a "rough" interface (with PVA and PMMA blending in G-16PVA) to a "smooth" one (with a relatively planar boundary between the densely packed PVA and the PMMA in G-32PVA). This finding (i.e. higher interfacial thermal conductance but lower interfacial affinity) agrees with a recent experimental report, ${ }^{67}$ which shows that the silicon/water interface has higher interfacial thermal conductance but lower interfacial affinity compared with the

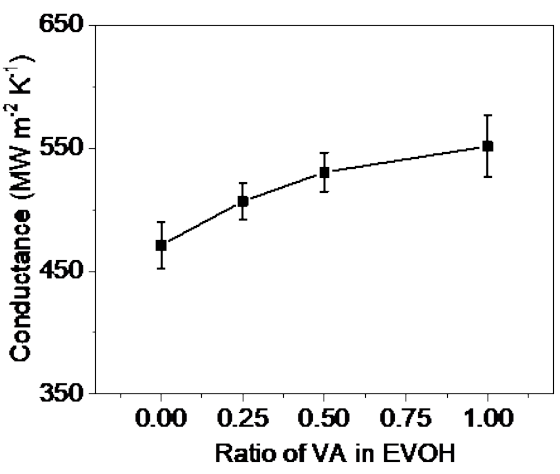

Fig. 9. Interfacial thermal conductance versus the number ratio of vinyl alcohol (VA) units in the copolymer of ethylene and vinyl alcohol (EVOH). Higher VA content means more hydrogen bonds.

graphene-coated silicon/water interface. Both studies indicate that the correlation between interfacial thermal conductance and interfacial adhesion is not universal. Interfacial adhesion is not necessarily an accurate indicator of interfacial thermal transport properties especially when the nanostructure fundamentally changes.

\subsection{Effect of hydrogen bonds density}

Compared with the pristine graphene/PMMA interface, the present hierarchical design with PVA is structurally different in two aspects: (1) it has backbone chains penetrating into the PMMA matrix; and (2) the backbone chains form hydrogen bonds in themselves and with PMMA. Both factors contribute to interfacial thermal transport.

To more directly reveal these contributions and the role of hydrogen bonds, PVA chains in G-32PVA were replaced by ethylene vinyl alcohol (EVOH) chains. PVA is a polymer of vinyl alcohol, while EVOH $\left(-\left[\left(\mathrm{C}_{2} \mathrm{H}_{4}\right)_{x}-\left(\mathrm{C}_{2} \mathrm{H}_{3} \mathrm{OH}\right)_{y}\right]_{m}\right)$ is a copolymer of vinyl alcohol and ethylene. Vinyl alcohol can form hydrogen bonds with PMMA, while ethylene cannot. Therefore, by changing $x$ and $y$, hydrogen bond density in the system is controllable. Four systems with different $\mathrm{EVOH}$ chains were analysed with $(x, y, m)=(0,1,8),(1,1,4),(3,1,2)$ and $(8,0,1)$. The first is PVA which has the most hydrogen bonds out of the four, and the last is polyethylene with no hydrogen bonds. The middle two have $50 \%$ and $25 \%$ maximum allowable hydrogen bonds, respectively, compared with PVA.

As shown in Fig. 9, interfacial thermal conductance increases with the hydrogen bond density. The contribution by the backbone can be found by comparing the polyethylene case with the pristine graphene, indicating an enhancement of 4.31 times. Graphene with PVA chains has interfacial thermal conductance $17.1 \%$ higher than that with polyethylene (PE) chains, which quantifies the effect of hydrogen bonds on top of the effect of the backbone structure. Furthermore, by comparing the autocorrelation of interfacial heat power for PVA-PMMA as shown in Fig. 7 and that for PE-PMMA in a previous study, ${ }^{26}$ we find that hydrogen bonds at the PVAPMMA interface enhances heat conducted through the more efficient thermal transport pathway, therefore further improving interfacial thermal conductance. 


\section{Conclusions}

This study investigates and designs hierarchically hydrogen bonded polymer chains as an extraordinary linker between graphene and the polymer matrix for enhanced energy transport across the interface. Compared with the pristine graphene/PMMA interface, graphene with 32 PVA chains grafted onto an area of $34.45 \AA \times 34.10 \AA$ is found to drastically enhance interfacial thermal conductance by a factor of 6.22 . The hierarchically hydrogen-bonded PVA decoration also outperforms other decorations with simple or no hydrogen bonds: (1) grafting two PVA chains on graphene improves interfacial thermal conductance more than grafting 14 hydroxyl groups in the same area; and (2) densely packed PVA decoration is $17.1 \%$ more effective than the polyethylene decoration with the same chain length and density. The outstanding thermal enhancement performance results from several structural and thermal mechanisms governed by the unique hydrogen bond structure. First, covalent grafting of PVA chains removes discontinuities in the temperature field. Second, due to strong hydrogen bonding with the matrix, grafted PVA chains are highly extended even at low grafting densities, effectively improving the along-chain conductivity of the linker. Third, the PVA decoration has much higher similarity with PMMA than graphene itself, making the decorated interface possess significantly improved vibrational coupling than its pristine counterpart. Due to the important role of hydrogen bonds in these enhancement mechanisms, interfacial thermal conductance is positively correlated with the hydrogen bond density in the hierarchical network. This study illustrates a new avenue to tune thermal transport performance at materials interfaces for thermal management and energy efficiency applications.

\section{Conflicts of interest}

The authors declare no competing financial interest.

\section{Acknowledgements}

This work is financially supported by CAREER Award No. CBET1751610 from the National Science Foundation. L.Z acknowledges the Dissertation Fellowship and the Graduate Research and Creative Opportunity Grant from Utah State University.

\section{Notes and references}

1. J. A. Tomko, A. Pena-Francesch, H. Jung, M. Tyagi, B. D. Allen, M. C. Demirel and P. E. Hopkins, Nature Nanotechnology, 2018, 13, 959-964.

2. N. Zhao, M. Yang, Q. Zhao, W. Gao, T. Xie and H. Bai, ACS Nano, 2017, 11, 4777-4784.

3. D. Ding, D. Wang, M. Zhao, J. Lv, H. Jiang, C. Lu and Z. Tang, Advanced Materials, 2017, 29, 1603444.
4. J. O. Tenorio-Pearl, E. D. Herbschleb, S. Fleming, C. Creatore, S. Oda, W. I. Milne and A. W. Chin, Nat Mater, 2017, 16, 208-213.

5. Y. Wang, Z. Qin, M. J. Buehler and Z. Xu, Nature Communications, 2016, 7, 12854.

6. A. E. Nel, L. Madler, D. Velegol, T. Xia, E. M. V. Hoek, P. Somasundaran, F. Klaessig, V. Castranova and M. Thompson, Nat Mater, 2009, 8, 543-557.

7. E. T. Swartz and R. O. Pohl, Reviews of Modern Physics, 1989, 61, 605-668.

8. M. Hu and D. Poulikakos, Nano Letters, 2012, 12, 5487-5494.

9. D. G. Cahill, P. V. Braun, G. Chen, D. R. Clarke, S. H. Fan, K. E. Goodson, P. Keblinski, W. P. King, G. D. Mahan, A. Majumdar, H. J. Maris, S. R. Phillpot, E. Pop and L. Shi, Applied Physics Reviews, 2014, 1, 011305.

10. Y. Liu, J. Huang, B. Yang, B. G. Sumpter and R. Qiao, Carbon, 2014, 75, 169-177.

11. T. Luo and J. R. Lloyd, Advanced Functional Materials, 2012, 22, 2495-2502.

12. Y. Wang, H. F. Zhan, Y. Xiang, C. Yang, C. M. Wang and Y. Y. Zhang, The Journal of Physical Chemistry C, 2015, 119, 12731-12738.

13. N. Mehra, L. Mu, T. Ji, X. Yang, J. Kong, J. Gu and J. Zhu, Applied Materials Today, 2018, 12, 92-130.

14. M. Hu, P. Keblinski and P. K. Schelling, Physical Review B, 2009, 79, 104305.

15. K. Gordiz and A. Henry, Scientific Reports, 2015, 5, 18361.

16. M. Hu and D. Poulikakos, International Journal of Heat and Mass Transfer, 2013, 62, 205-213.

17. S. C. Lin and M. J. Buehler, Nanotechnology, 2013, 24, 165702.

18. Y. Wang, C. H. Yang, Y. W. Mai and Y. Y. Zhang, Carbon, 2016, 102, 311-318.

19. M. D. Losego, M. E. Grady, N. R. Sottos, D. G. Cahill and P. V. Braun, Nature Materials, 2012, 11, 502.

20. P. J. O'Brien, S. Shenogin, J. X. Liu, P. K. Chow, D. Laurencin, P. H. Mutin, M. Yamaguchi, P. Keblinski and G. Ramanath, Nature Materials, 2013, 12, 118-122.

21. S. Kaur, N. Raravikar, B. A. Helms, R. Prasher and D. F. Ogletree, Nature Communications, 2014, 5, 4082.

22. T. Jiang, X. Zhang, S. Vishwanath, X. Mu, V. Kanzyuba, D. A. Sokolov, S. Ptasinska, D. B. Go, H. G. Xing and T. Luo, Nanoscale, 2016, 8, 10993-11001.

23. S. Majumdar, J. A. Sierra-Suarez, S. N. Schiffres, W. L. Ong, C. F. Higgs, A. J. H. McGaughey and J. A. Malen, Nano Letters, 2015, 15, 2985-2991.

24. Y. Wang, C. Yang, Q.-X. Pei and Y. Zhang, ACS Applied Materials \& Interfaces, 2016, 8, 8272-8279.

25. Y. Wang, H. F. Zhan, Y. Xiang, C. Yang, C. M. Wang and Y. Y. Zhang, J Phys Chem C, 2015, 119, 12731-12738.

26. L. Zhang and L. Liu, ACS Applied Materials \& Interfaces, 2017, 9, 28949-28958.

27. L. Zhang, Z. Bai and L. Liu, Advanced Materials Interfaces, 2016, 3, 1600211 .

28. T. Zhang, A. R. Gans-Forrest, E. Lee, X. Q. Zhang, C. Qu, Y. S. Pang, F. Sun and T. F. Luo, Acs Applied Materials \& Interfaces, 2016, 8, 33326-33334.

29. N. Mehra, M. A. Kashfipour and J. Zhu, Applied Materials Today, 2018, 13, 207-216. 
30. L. Mu, J. He, Y. Li, T. Ji, N. Mehra, Y. Shi and J. Zhu, The Journal of Physical Chemistry C, 2017, 121, 14204-14212.

31. G.-H. Kim, D. Lee, A. Shanker, L. Shao, M. S. Kwon, D. Gidley, J. Kim and K. P. Pipe, Nature Materials, 2014, 14, 295.

32. M. Fang, K. Wang, H. Lu, Y. Yang and S. Nutt, Journal of Materials Chemistry, 2009, 19, 7098-7105.

33. M. Fang, K. Wang, H. Lu, Y. Yang and S. Nutt, Journal of Materials Chemistry, 2010, 20, 1982-1992.

34. M. Steenackers, A. M. Gigler, N. Zhang, F. Deubel, M. Seifert, L. H. Hess, C. H. Y. X. Lim, K. P. Loh, J. A. Garrido and R. Jordan, Journal of the American Chemical Society, 2011, 133, 1049010498.

35. H. Kim, S. Kobayashi, M. A. AbdurRahim, M. J. Zhang, A. Khusainova, M. A. Hillmyer, A. A. Abdala and C. W. Macosko, Polymer, 2011, 52, 1837-1846.

36. H. J. Salavagione, Journal of Materials Chemistry A, 2014, 2, 71387146.

37. Z. Liu, J. T. Robinson, X. Sun and H. Dai, Journal of the American Chemical Society, 2008, 130, 10876-10877.

38. H. J. Salavagione, M. A. Gomez and G. Martínez, Macromolecules, 2009, 42, 6331-6334.

39. K. Binder, Monte Carlo and Molecular Dynamics Simulations in Polymer Science, Oxford University Press, New York and Oxford, 1995.

40. W. Humphrey, A. Dalke and K. Schulten, Journal of molecular graphics, 1996, 14, 33-38.

41. S. Plimpton, J Comput Phys, 1995, 117, 1-19.

42. W. L. Jorgensen, D. S. Maxwell and J. Tirado-Rives, J. Am. Chem. Soc., 1996, 118, 11225-11236.

43. M. L. P. Price, D. Ostrovsky and W. L. Jorgensen, J. Comput. Chem., 2001, 22, 1340-1352.

44. P. Lazar, F. Karlicky, P. Jurecka, M. Kocman, E. Otyepkova, K. Safarova and M. Otyepka, J. Am. Chem. Soc., 2013, 135, 63726377.

45. L. J. Fetters, D. J. Lohse, D. Richter, T. A. Witten and A. Zirkel, Macromolecules, 1994, 27, 4639-4647.

46. F. MullerPlathe, Journal of Chemical Physics, 1997, 106, 60826085.

47. C. Nieto-Draghi and J. B. Avalos, Mol. Phys., 2003, 101, 23032307.

48. T. Schneider and E. Stoll, Phys Rev B, 1978, 17, 1302-1322.

49. M. Assael, K. Antoniadis and J. Wu, Int J Thermophys, 2008, 29, 1257-1266.

50. H. Chen, V. V. Ginzburg, J. Yang, Y. Yang, W. Liu, Y. Huang, L. Du and B. Chen, Progress in Polymer Science, 2016, 59, 41-85.

51. T. Zhang and T. Luo, The Journal of Physical Chemistry B, 2016, 120, 803-812.

52. S. Shen, A. Henry, J. Tong, R. Zheng and G. Chen, Nat Nano, 2010, 5, 251-255.

53. A. Henry and G. Chen, Physical Review Letters, 2008, 101, 235502.

54. F. Schreiber, A. Eberhardt, T. Y. B. Leung, P. Schwartz, S. M. Wetterer, D. J. Lavrich, L. Berman, P. Fenter, P. Eisenberger and G. Scoles, Physical Review B, 1998, 57, 12476-12481.

55. G. Poirier and E. Pylant, Science, 1996, 272, 1145-1148.

56. P. H. Hermans, J. J. Hermans, D. Vermaas and A. Weidinger, Journal of Polymer Science, 1948, 3, 1-9.
57. G. A. Miño-Galaz and G. Gutierrez, Chemical Physics Letters, 2015, 635, 16-22.

58. G. Miño, R. Barriga and G. Gutierrez, The Journal of Physical Chemistry B, 2014, 118, 10025-10034.

59. L. Zhang, M. Ruesch, X. Zhang, Z. Bai and L. Liu, RSC Advances, 2015, 5, 87981-87986.

60. L. Zhang, T. Chen, H. Ban and L. Liu, Nanoscale, 2014, 6, 77867791.

61. L. Zhang, Z. Bai, H. Ban and L. Liu, Physical Chemistry Chemical Physics, 2015, 17, 29007-29013.

62. B. Li, J. Lan and L. Wang, Physical Review Letters, 2005, 95, 104302.

63. Y. Wang and P. Keblinski, Applied Physics Letters, 2011, 99, 073112.

64. N. Shenogina, R. Godawat, P. Keblinski and S. Garde, Physical Review Letters, 2009, 102, 156101.

65. Z. Ge, D. G. Cahill and P. V. Braun, Physical Review Letters, 2006, 96, 186101.

66. H. F. Zhan, G. Zhang, V. B. C. Tan, Y. Cheng, J. M. Bell, Y. W. Zhang and Y. T. Gu, Advanced Functional Materials, 2016, 26, 52795283.

67. B. Ramos-Alvarado, S. Kumar and G. P. Peterson, The Journal of Physical Chemistry Letters, 2016, 7, 3497-3501. 


\title{
Supporting Information
}

\section{Hierarchically Hydrogen-Bonded Graphene/Polymer}

\author{
Interfaces with Drastically Enhanced Interfacial
}

\section{Thermal Conductance}

Lin Zhang, Ling Liu*

Department of Mechanical and Aerospace Engineering, Utah State University, Logan, UT

84322, USA.*E-mail: ling.liu@usu.edu 


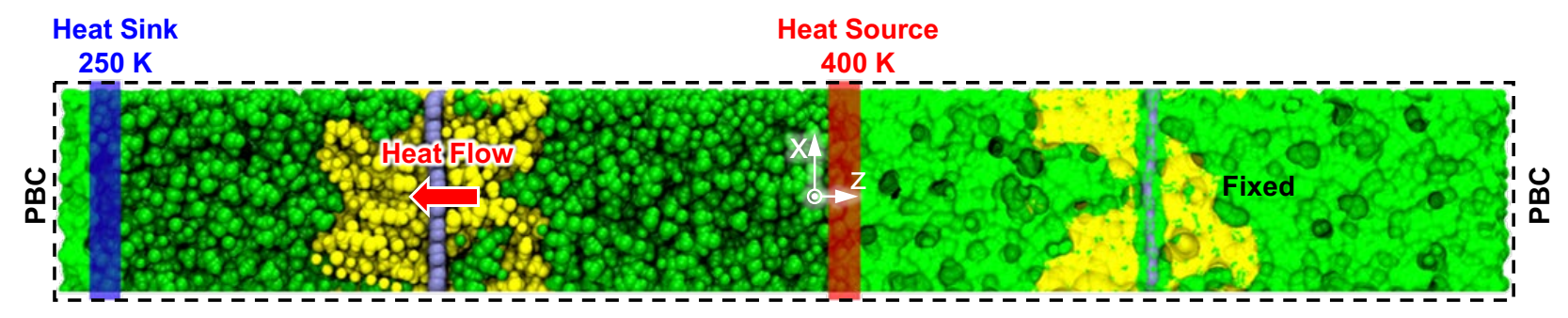

Fig. S1. System setup for nonequilibrium molecular dynamics (NEMD) simulations (green: PMMA; yellow: PVA; blue: graphene). Atoms in the frozen region were fixed. Temperatures of the heat source and the heat sink were fixed at $400 \mathrm{~K}$ and $250 \mathrm{~K}$, respectively. Periodic boundary conditions (PBC) were applied in all three directions.

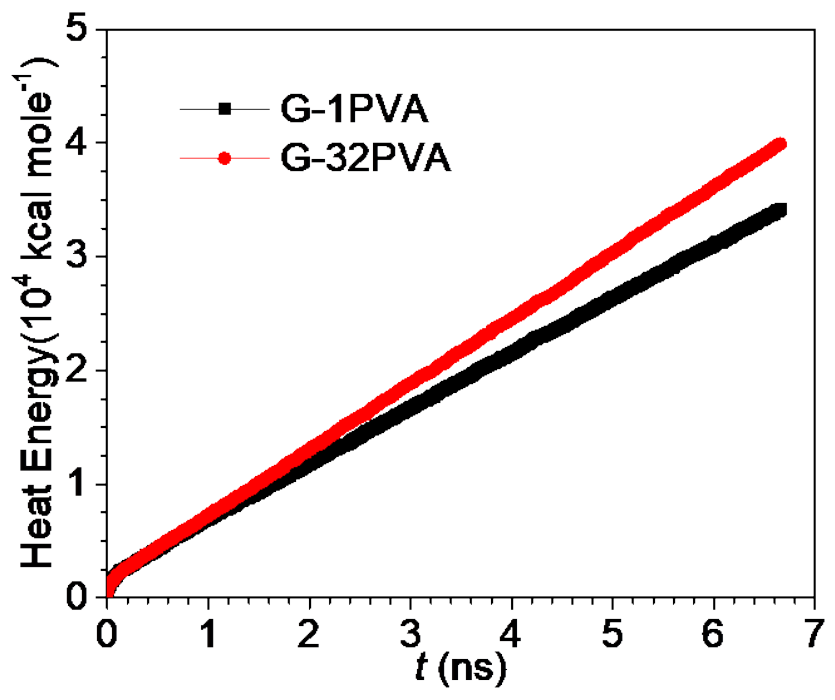

Fig. S2. Heat energy added to the heat source by the Langevin thermostat versus time in NEMD. 


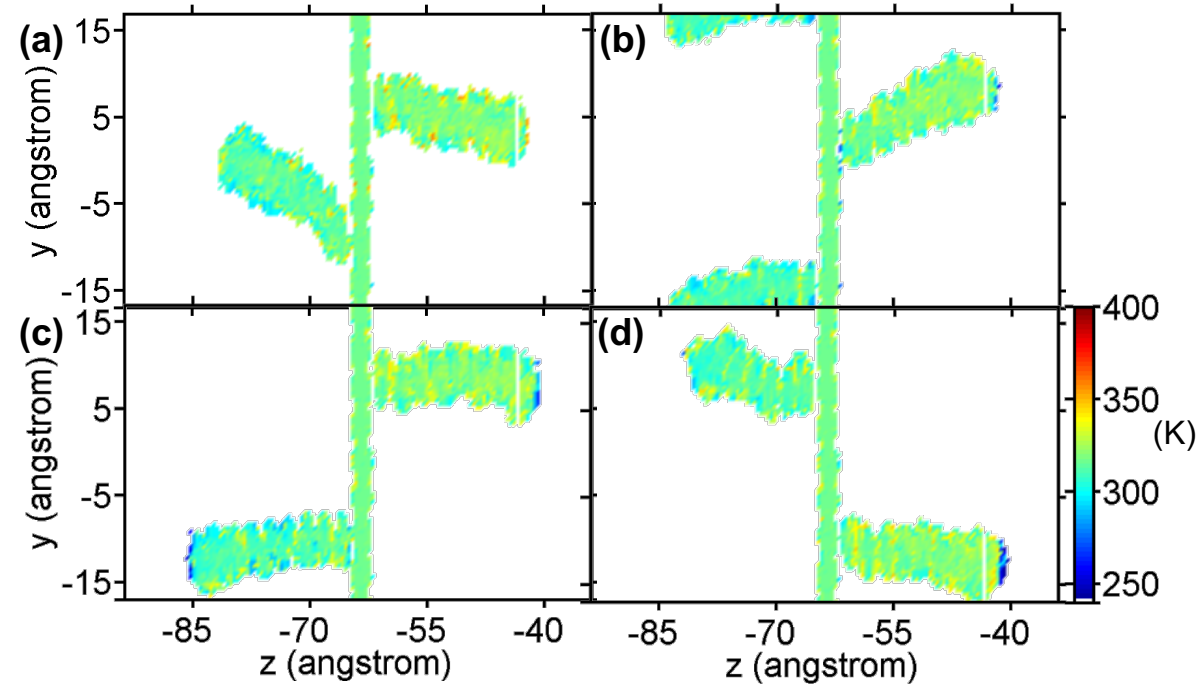

Fig. S3. Temperature contours of graphene and a pair of PVA chains in G-32PVA. PVA chains shown in (a)-(d) were randomly chosen for a total of 32 pairs. Distance between the grafting points of two PVA chains in a pair is comparable to that in G-1PVA.

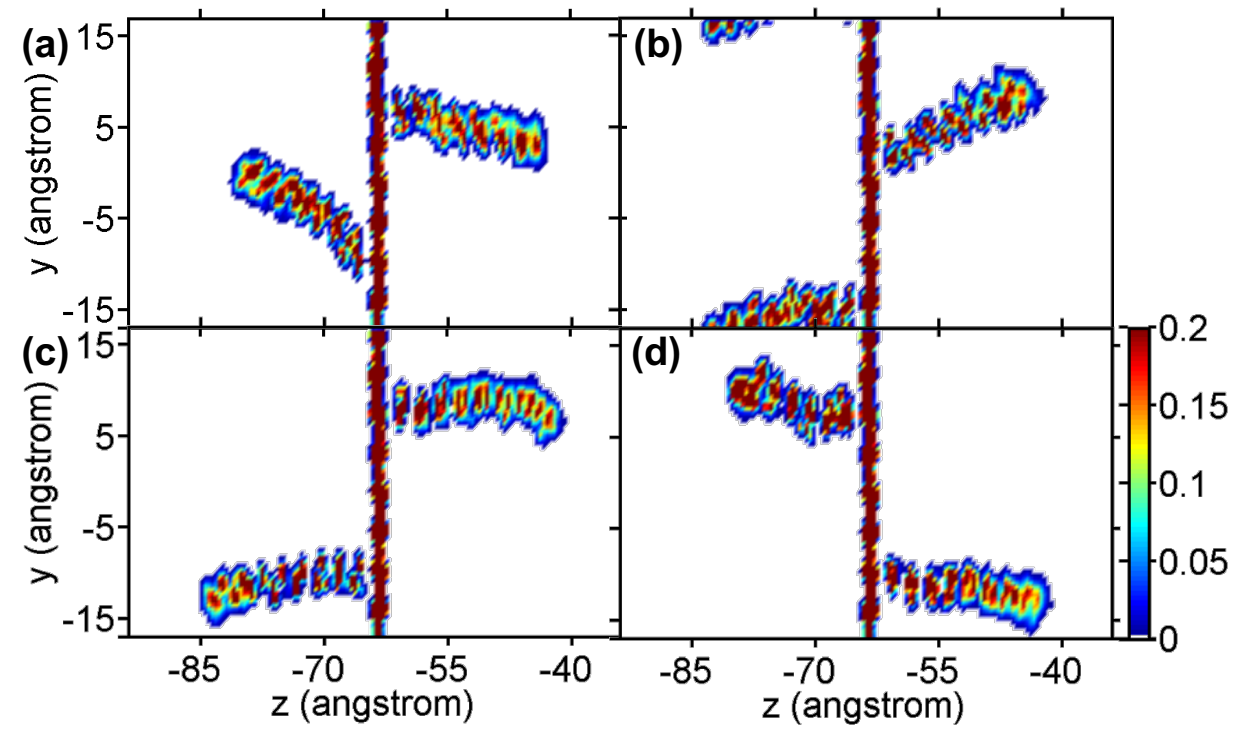

Fig. S4. Contour plots of the atomic number density of graphene and selected pairs of PVA chains in G-32PVA. 


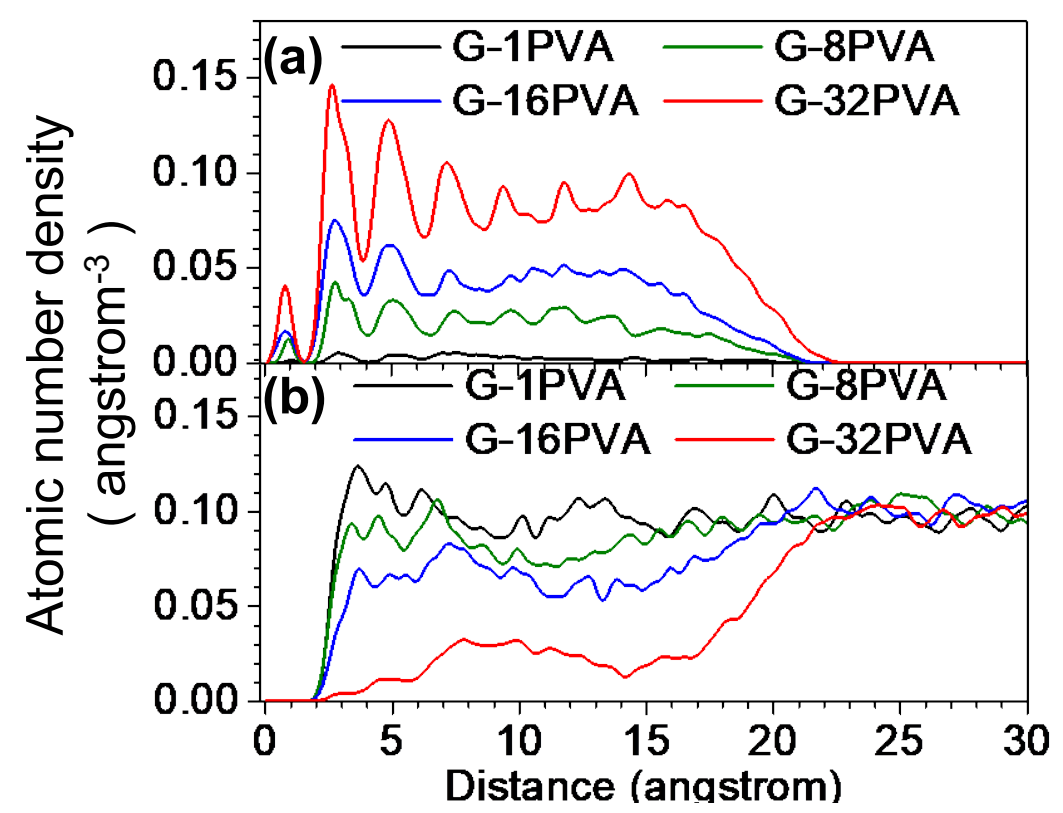

Fig. S5. Axial atomic number density distributions of (a) PVA and (b) PMMA along the direction perpendicular to the surface of graphene. Atoms were binned into cells of $0.2 \AA$ along the $z$-direction starting from the surface of graphene. Over 8000 frames were analyzed and averaged. PVA in G-32PVA shows long-range ordering until $16 \AA$ away from the graphene surface. This distance is very close to the length of a relaxed straight PVA chain employed for this study (19.9 $\AA$ ), proving the highly extended configuration of PVAs in G-32PVA. With less PVA chains in the system, the ordering length is only slightly reduced, suggesting extended configurations even at low grafting densities. 

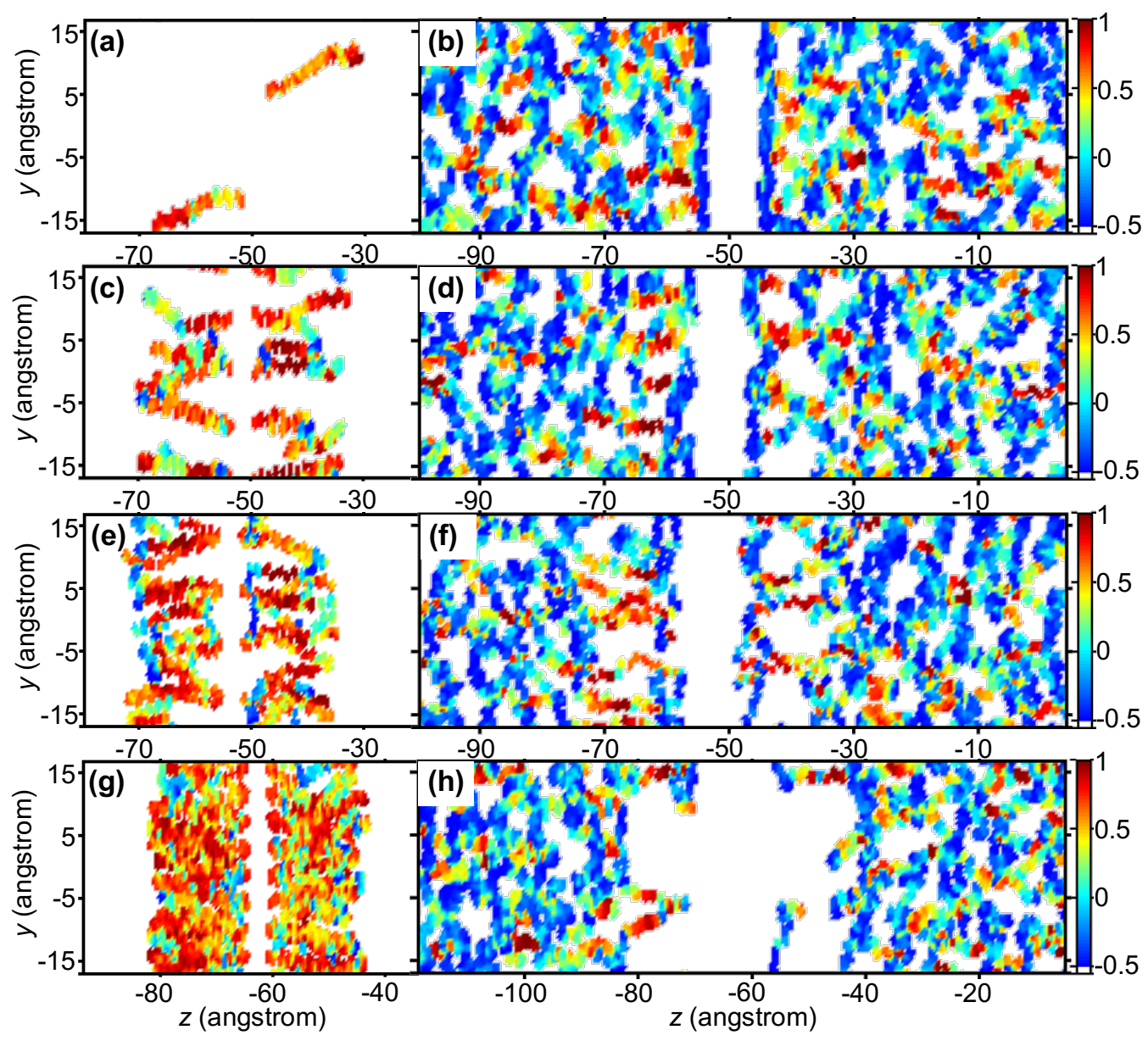

Fig. S6. Local Herman's orientation for polymer main chains in G-XPVA at different PVA grafting densities: (a, c, e, g) PVA and (b, d, f, h) PMMA. From top to bottom: $X=1,8,16$ and 32. 


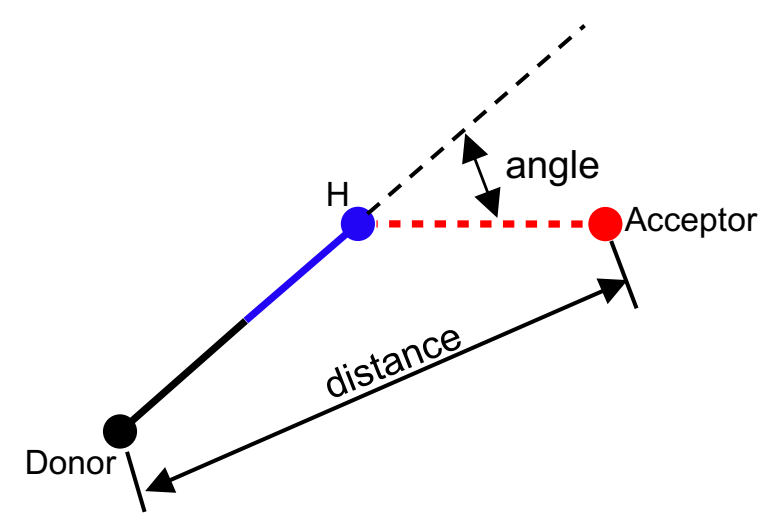

Fig. S7. Geometric criteria used to identify a hydrogen bond: (1) the distance between the hydrogen bond donor (D) and the acceptor (A) is smaller than $3.0 \AA$; and (2) the angle of D-H (hydrogen)-A is smaller than $25^{\circ}$.

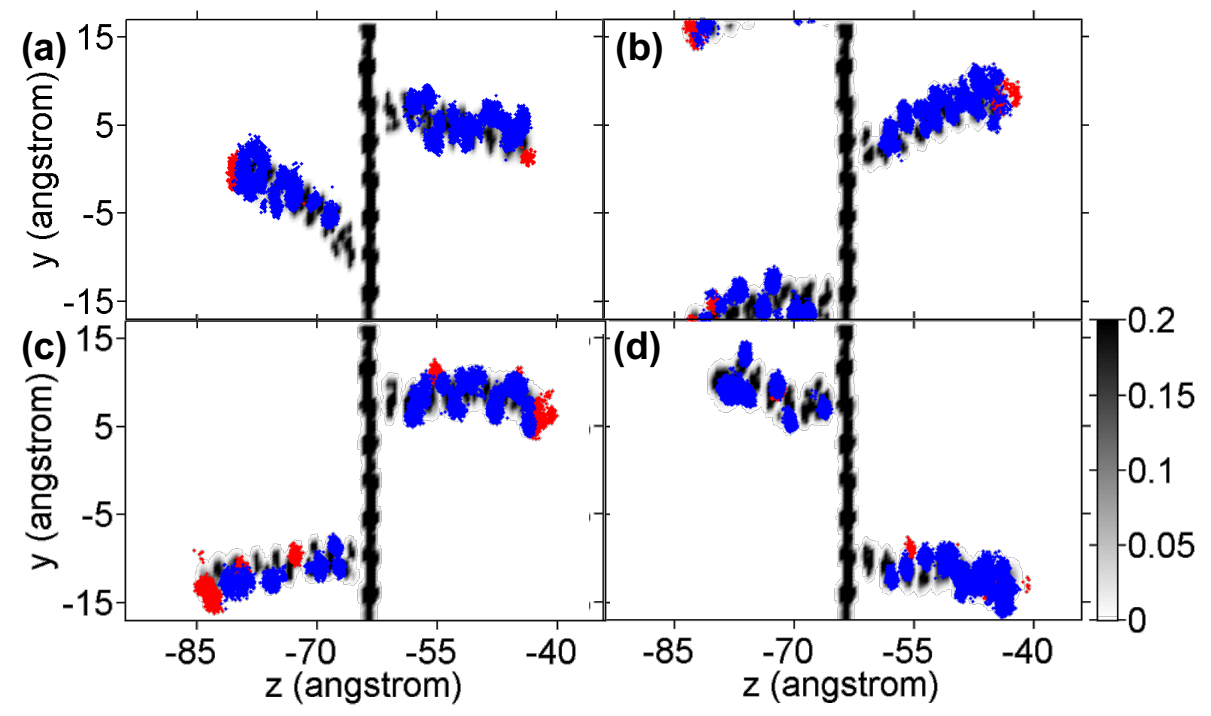

Fig. S8. Hydrogen bonds formed in four selected pairs of PVA chains in G-32PVA. Grey color dots show the atomic number density. Red dots show hydrogen bonds formed between PVA and PMMA, while blue dots show those formed between and within PVA chains. 

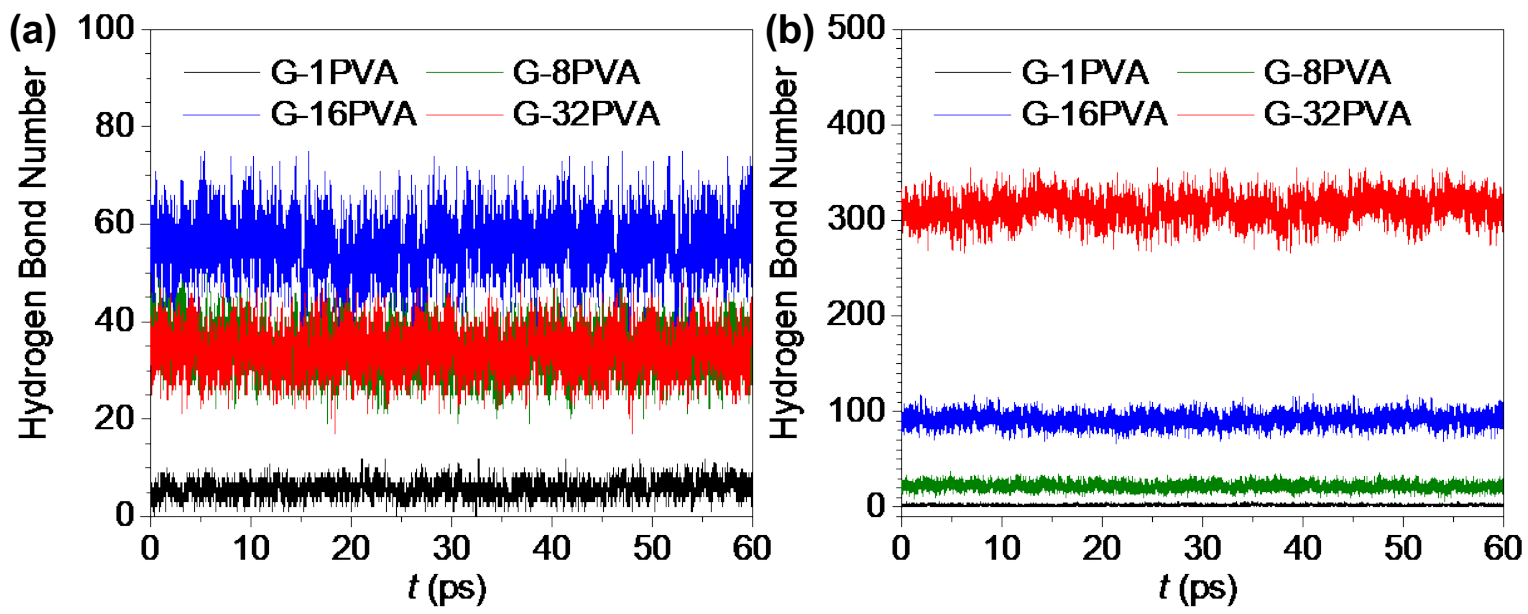

Fig. S9. Number of hydrogen bonds versus time: (a) hydrogen bonds between PVA and PMMA and (b) hydrogen bonds between and within PVA chains.

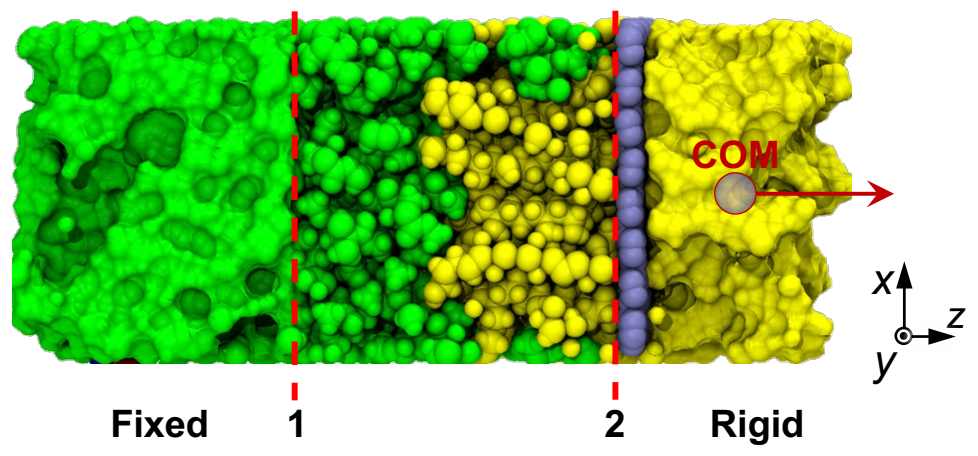

Fig. S10. An atomistic model for MD simulation of the pull-out process. Atoms of PMMA (green) to the left of plane "1" are fixed. Atoms of PVA (yellow) to the right of plane " 2 " are fixed. Atoms of graphene (ice blue) and those between "1" and "2" are movable. The center of mass (COM) of graphene and the rigid part of PVA as a whole is moved at a constant velocity along the $+z$ direction. The simulation was performed in the NVT ensemble with the time step of $0.1 \mathrm{fs}$. The pulling force was sampled at different separations. Work of adhesion was evaluated by integrating the force-displacement curve. 


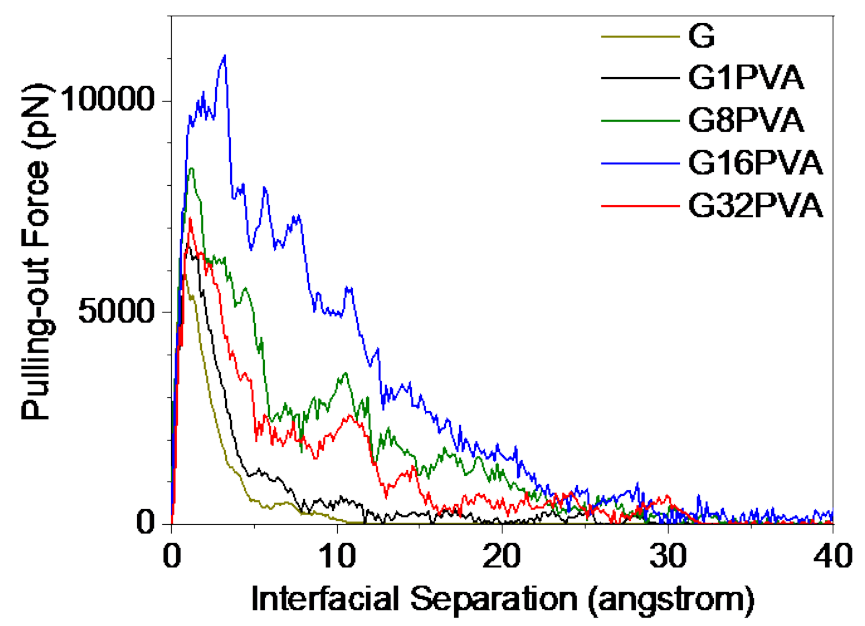

Fig. S11. Pull-out force versus interfacial separation for G-XPVA with different PVA grafting densities. G represents pristine graphene/PMMA interface. 